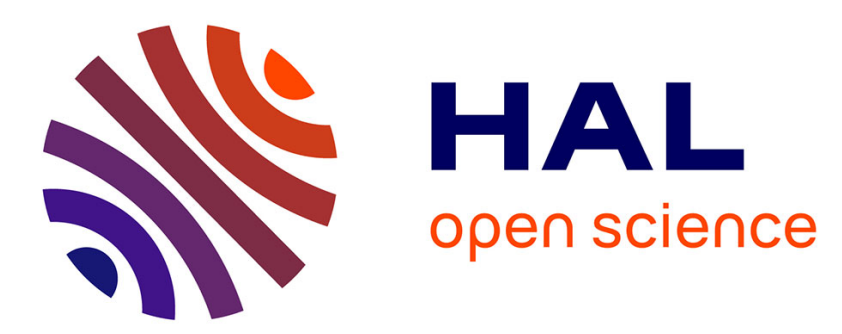

\title{
Lagrangian and spectral analysis of the forced flow past a circular cylinder using pulsed tangential jets
}

Thierry Jardin, Yannick Bury

\section{To cite this version:}

Thierry Jardin, Yannick Bury. Lagrangian and spectral analysis of the forced flow past a circular cylinder using pulsed tangential jets. Journal of Fluid Mechanics, 2012, vol. 696, pp. 285-300. 10.1017/jfm.2012.35 . hal-00739941

\section{HAL Id: hal-00739941 \\ https://hal.science/hal-00739941}

Submitted on 9 Oct 2012

HAL is a multi-disciplinary open access archive for the deposit and dissemination of scientific research documents, whether they are published or not. The documents may come from teaching and research institutions in France or abroad, or from public or private research centers.
L'archive ouverte pluridisciplinaire $\mathbf{H A L}$, est destinée au dépôt et à la diffusion de documents scientifiques de niveau recherche, publiés ou non, émanant des établissements d'enseignement et de recherche français ou étrangers, des laboratoires publics ou privés. 


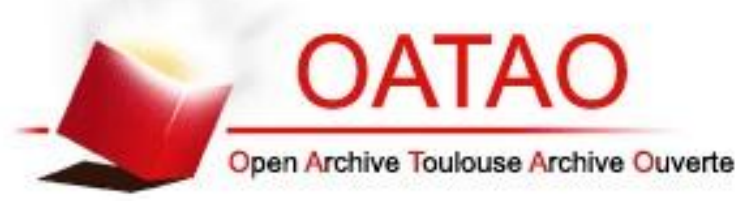

\section{Open Archive Toulouse Archive Ouverte (OATAO)}

OATAO is an open access repository that collects the work of Toulouse researchers and makes it freely available over the web where possible.

This is an author-deposited version published in: http://oatao.univ-toulouse.fr/ Eprints ID: 6502

To link to this article: DOI: $10.1017 / \mathrm{jfm} .2012 .35$

URL: http://dx.doi.org/10.1017/jfm.2012.35

To cite this version: Jardin, Thierry and Bury, Yannick Lagrangian and spectral analysis of the forced flow past a circular cylinder using pulsed tangential jets. (2012) Journal of Fluid Mechanics, vol. 696. pp. 285-300. ISSN 0022-1120

Any correspondence concerning this service should be sent to the repository administrator: staff-oatao@inp-toulouse.fr 


\title{
Lagrangian and spectral analysis of the forced flow past a circular cylinder using pulsed tangential jets
}

\author{
T. Jardin and Y. Bury $\dagger$ \\ Université de Toulouse, ISAE, 31055 Toulouse, France \\ (Received 19 May 2011; revised 8 December 2011; accepted 13 January 2012; \\ first published online 1 March 2012)
}

We numerically investigate the influence of pulsed tangential jets on the flow past a circular cylinder. To this end a spectral-Lagrangian dual approach is used on the basis of time-series data. The analysis reveals that the flow response to unsteady forcing is driven by strong interactions between shear layers and pulsed jets. The latter preferentially lead to either the lock-on regime or the quasi-steady vortex feeding regime whether the excitation frequency is of the order of, or significantly greater than, the frequency of the natural instability. The intensity of the wake vortices is mainly influenced by the momentum coefficient through the introduction of opposite-sign vorticity in the shear layers. This feature is emphasized using a modal-based time reconstruction, i.e. by reconstructing the flow field upon a specific harmonic spectrum associated with a characteristic time scale. The quasi-steady regime exhibits smallscale counter-rotating vortices that circumscribe the separated region. In the lock-on regime, atypical wake patterns such as $2 \mathrm{P}$ or $\mathrm{P}+\mathrm{S}$ can be observed, depending on the forcing frequency and the momentum coefficient, highlighting remarkable analogies with oscillating cylinders.

Key words: flow control, vortex flows, wakes

\section{Introduction}

During the past few decades, synthetic jets have proven to be efficient tools for controlling the wake of shaped and bluff bodies (Glezer \& Amitay 2002). For instance, it is now established that adding momentum to a separated boundary layer can lead to flow reattachment, resulting in lift enhancement (Post \& Corke 2004), reduction of airframe noise (Thomas, Kozlov \& Corke 2008) or stabilization of slender forebodies (Liu et al. 2008). The efficiency of such devices (as opposed to passive appendices such as vortex generators) relies on their ability to provide unsteady actuation and to modulate specific flow frequencies.

The determination of these frequencies is a complex issue owing to the various flow instabilities, which are strongly Reynolds-number dependent, and their potential coupling. Although it has been shown that operating at dominant flow frequencies can lead to a significant impact on the flow, the identification of the underlying physical mechanisms still remains a challenge. This leads us to analyse the interplay between 
the flow and the vortical structures generated by the pulsed jets' unsteady actuation. This strategy (instability manipulation through vortex/shear layer interaction) primarily comes within the framework of direct wake control, as opposed to boundary layer control used to delay separation by triggering transition to turbulence and enhancing near-wall momentum (Choi, Jeon \& Kim 2008).

This paper is focused on the modification of a two-dimensional circular cylinder wake by unsteady surface tangential jets - such as those induced by the use of surface dielectric barrier discharge (SDBD) plasma actuators - at Reynolds number 1000. Although the present study relies on two-dimensional computations, and the flow past a circular cylinder at Reynolds number 1000 is three-dimensional, the forcing is expected to promote two-dimensional flow in the close wake of the cylinder and to highlight the physics driving the flow response to control vortices/shear layer interaction.

We investigate time-series data using a spectral analysis, derived from the Arnoldi method as recently introduced by Schmid \& Sesterhenn (2008), combined with a Lagrangian method based on vortex tracking. This approach enables a thorough comparison between three distinct flow cases: the unforced flow, the quasi-steadyregime controlled flow at forcing frequency $S t_{\text {jet }}=4$, and the lock-on-regime controlled flow at $S t_{j e t}=0.5$. Each case is characterized by specific interactions driving the flow response to the actuation. The Lagrangian approach is employed to decipher the flow changes in terms of vortical activity. It shows how the tangential jets modify the von Kármán vortex dynamics through their strong interaction with shear layers. The various modes resulting from these interactions are clearly revealed by the spectral analysis. Based on this spectral analysis, the mode-based temporal reconstruction of the flow gives access to the rotational contribution of each time scale. Finally the analysis is extended to additional flow control cases.

\section{Numerical methods}

The two-dimensional time-dependent Navier-Stokes equations around a circular cylinder of diameter $D$ are directly solved using an Eulerian finite-volume method. The Reynolds number $R e$ based on $D$ and on the free-stream velocity $U_{\infty}$ is set to 1000. Assuming incompressible flow, the equations read:

$$
\begin{gathered}
\nabla \cdot \boldsymbol{v}=0 \\
\frac{\partial \boldsymbol{v}}{\partial t}+(\boldsymbol{v} \cdot \nabla) \boldsymbol{v}=-\frac{1}{\rho} \nabla p+v \nabla^{2} \boldsymbol{v}+\boldsymbol{S}_{j e t} .
\end{gathered}
$$

Here $v$ is the velocity, $p$ the pressure, $\rho$ and $v$ the fluid density and kinematic viscosity respectively. $\boldsymbol{S}_{\text {jet }}$ is a body force source term used to model tangential jet actuators of width $10^{\circ}$ and height $D / 50$ at the surface of the cylinder. The actuators are centred at an angle $\theta_{j e t}=110^{\circ}$ from the streamwise axis (figure $1 b$ : for the sake of clarity, the actuator meshing is not depicted here), which is near the mean separation point where the forcing is found to be the most effective. Note that this observation corroborates experimental results from Jukes \& Choi (2009), obtained on a plasmacontrolled circular cylinder in the subcritical regime. The tangential jets are driven by a pulsed signal at a non-dimensional frequency $S t_{j e t}=f_{j e t} D / U_{\infty}$ and a force coefficient $C_{j e t}=2 \lambda_{\text {jet }} S_{\text {jet }} / \rho U_{\infty}^{2} D$, where $\lambda_{\text {jet }}$ is the duty cycle of the actuators (i.e. the ratio between the time during which they are active and the total forcing period $\left.1 / S t_{j e t}\right)$. 
(a)

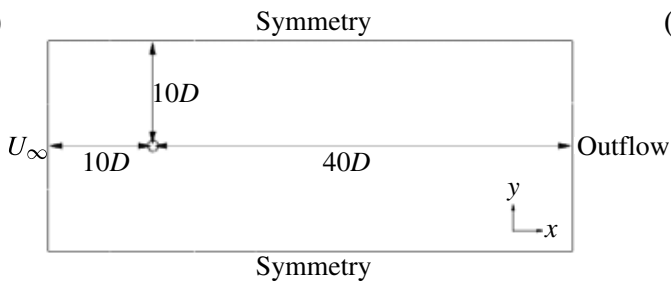

(b)

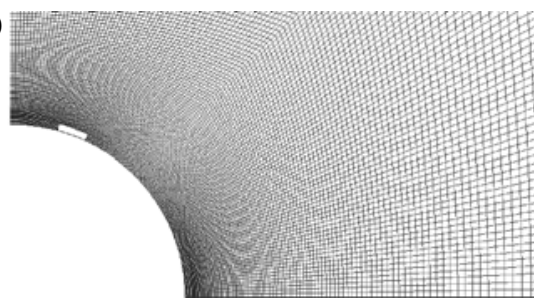

FIGURE 1. Computational domain (a) and close-up view of the mesh (b).

The body force source term is expressed as:

$$
S_{j e t}= \begin{cases}C_{j e t} \times \rho U_{\infty}^{2} D & \text { for } t \in\left[0 ; \lambda_{j e t} / S t_{j e t}[\right. \\ 0 & \text { for } t \in\left[\lambda_{\text {jet }} / S t_{j e t} ; 1 / S t_{j e t}[.\right.\end{cases}
$$

Unless otherwise specified, $\lambda_{\text {jet }}$ and $C_{j e t}$ are fixed to 0.5 and 0.067 respectively. Hereafter (2.1a) and (2.1b) will be defined in Cartesian coordinates $(x, y)$.

The computational domain is shown in figure 1 . The inlet boundary is located 10 diameters upstream the cylinder and is subjected to a velocity Dirichlet condition. A zero-diffusion-flux condition is prescribed at the outlet boundary located 40 diameters downstream the cylinder. The upper and lower boundaries are defined as symmetric boundaries and the cylinder surface is modelled as a non-slip surface. The domain is composed of 120000 cells.

The spatial and temporal discretizations are achieved using second-order upwind schemes and a second-order implicit time stepping method respectively. The pressure-velocity coupling is obtained using a SIMPLE algorithm. Approximately 280 time steps are performed within one shedding period, satisfying the CFL condition (Courant number close to unity).

Further simulations were carried out to ensure that the results are independent of the number of cells, the time step and the position of the external boundary conditions. In addition, the mean drag coefficient $C_{D}$ and the Strouhal number $S t$ obtained at Reynolds number 1000 accurately match two-dimensional computational results reported in the literature (Henderson 1997), with $C_{D}=1.56$ and $S t=0.24$.

\section{Flow analysis methods}

The spatio-temporal evolution of the near- and far-wake vortices and the modal signature of the flow are evaluated using a spectral analysis derived from the Arnoldi method combined with a vortex-tracking method.

\subsection{Dynamic mode decomposition}

The spectral analysis employed here has recently been proposed by Schmid \& Sesterhenn (2008) as a convenient tool to extract instabilities from a given timeresolved snapshot sequence of the flow in a spatial domain $\Omega$. For a sufficient number $N$ of snapshots separated by a constant time interval $\Delta t$, the flow field at time $(N+1) \times \Delta t$ can be faithfully expressed as a linear combination of the previous flow fields (snapshots from time $t=1$ to time $t=N \times \Delta t$ ). Consider two matrices $\boldsymbol{V}_{1}^{N}$ and $\boldsymbol{V}_{2}^{N+1}$ resulting from the concatenation of $N$ sets of flow velocity measurements $\boldsymbol{V}(\Omega, t)$ obtained from time $t=1$ to time $t=N \times \Delta t$ and from time $t=2$ to time $t=(N+1) \times \Delta t$ respectively. The coefficients of the linear combination 
are stacked in a companion matrix $\boldsymbol{C}$ such that:

$$
\boldsymbol{V}_{2}^{N+1} \approx \boldsymbol{V}_{1}^{N} \boldsymbol{C}
$$

where the eigenvalues $\lambda_{i}$ of $\boldsymbol{C}$ are approximations to some of the eigenvalues (Ritz values) of a higher-dimension inter-snapshot linear map. The resulting dynamic modes (or Koopman modes) can be expressed as:

$$
\phi_{i}=\boldsymbol{V}_{1}^{N} v_{i}
$$

where $v_{i}$ is the $i$ th eigenvector of $\boldsymbol{C}$. The magnitude and frequency of the dynamic mode $\phi_{i}$ are defined as $\left\|\phi_{i}\right\|$ and $\omega_{i}=\operatorname{Im}\left\{\log \left(\lambda_{i}\right)\right\} / 2 \pi \Delta t$ respectively. A more thorough description of the theoretical basis can be found in Rowley et al. (2009) and Schmid (2010).

This method, referred to as the dynamic mode decomposition (DMD), has successively been applied to image-based flow visualization of a laminar axisymmetric jet (Schmid 2009), lid-driven cylindrical cavity particle image velocimetry (PIV) measurements (Schmid, Meyer \& Pust 2009), jet in cross-flow direct numerical simulation (DNS) data (Rowley et al. 2009) or transitional water jet tomo-PIV measurements (Schmid et al. 2010). See also Schmid (2010) for further applications. In particular, Rowley et al. (2009) demonstrated that whereas a single proper orthogonal decomposition (POD) mode may be associated with more than one frequency, a DMD mode is exclusively linked to one given frequency. The interest for unsteady flow control applications is hence straightforward since DMD has the capability to isolate a specific time scale, allowing, for instance, the characterization of the flow dynamics associated with the actuator by knowing its actuation frequency.

On the basis of these dynamic modes, most of the initial flow can be reconstructed using only a few dominant modes, following relation:

$$
V_{\text {rec }}(\Omega, t)=\sum_{i=1}^{L<N} v_{i}^{-1}(t) \phi_{i}
$$

where $V_{\text {rec }}(\Omega, t)$ is the reconstructed flow field based on $L$ modes. For periodic flows, the dynamic modes can be organized in terms of fundamental Fourier modes and their harmonics, since the Koopman modes reduce to a discrete temporal Fourier transform (Rowley et al. 2009). Each fundamental mode $k$, associated with a specific time scale $1 / \omega_{k}$, and its harmonics constitute a subspace, referred to as a harmonic spectrum and denoted $\zeta^{k}$. In contrast to POD, it is thus possible to retrieve the modal contribution of a specific time scale to the initial flow by summing $i$ upon a specific harmonic spectrum $\zeta^{k}$.

\subsection{Vortex tracking}

An automatic vortex core identification and tracking algorithm (detailed in Bury, Morton \& Charles 2008) is used to objectively and accurately determine the characteristics and dynamics of the vortical structures: core position, size and intensity evolution. Based on the $\lambda_{2}$-criterion (Jeong \& Hussain 1995), the vortex core $\Sigma$ is identified and relevant parameters are computed as follows:

$$
\Gamma=\iint_{\Sigma} \omega \mathrm{d} x \mathrm{~d} y
$$

where $\omega$ refers to the vorticity field distribution in the core, and $\Gamma$ is the vortex circulation. 
The position $\left(x_{c}, y_{c}\right)$ of the vortex centroid is then given by:

$$
\begin{aligned}
& x_{c}=\frac{1}{\Gamma} \iint_{\Sigma} x \omega \mathrm{d} x \mathrm{~d} y, \\
& y_{c}=\frac{1}{\Gamma} \iint_{\Sigma} y \omega \mathrm{d} x \mathrm{~d} y .
\end{aligned}
$$

The definition of the vortex radius $R$ is based on polar moments of vorticity:

$$
R=\left[\frac{1}{2 \Gamma} \iint_{\Sigma}\left[\left(x-x_{c}\right)^{2}+\left(y-y_{c}\right)^{2}\right] \omega \mathrm{d} x \mathrm{~d} y\right]^{1 / 2}
$$

Finally the tangential velocity $V_{\theta}$ at the periphery of the vortex core is defined as:

$$
V_{\theta}=\frac{\Gamma}{2 \pi R}
$$

Henceforth all parameters will be normalized with respect to $D$ and $U_{\infty}$. Normalization will be indicated by superscript *

\section{Spatial-domain analysis}

In this section we focus on the spatial-domain analysis of the unforced flow and two forced cases, corresponding to $S t_{j e t}=4$ and $S t_{j e t}=0.5$ respectively. A qualitative description of the wake pattern is conducted on the basis of instantaneous vorticity field visualizations (figure 2). Quantitative analysis is carried out via the dynamics of the vortices identified from the vortex tracking algorithm (figure 3). This approach delineates three regions separated by discontinuities in the spatio-temporal evolution of the vortex parameters. This partitioning, respectively characterizing the vortices' formation, shedding and far-wake advection, provides key features for a thorough comparison between forced and unforced flow fields around the cylinder.

\subsection{Unforced and $S t_{j e t}=4$ forced flows}

The unforced and $S t_{\text {jet }}=4$ forced flows converge to a periodic state associated with von Kármán vortex shedding. In both cases, vortex shedding is promoted by the occurrence of cross-wake interaction between lower and upper vortices, concomitantly with vorticity saturation in the unforced case (Gerrard 1966; Williamson 1996). Yet, while the unforced case is characterized by laminar boundary layer separation and its alternating rolling up into counter-rotating vortices that are shed and advected downstream, the $S t_{\text {jet }}=4$ forced flow case exhibits smaller-scale vortices that are advected along the shear layer before merging into the von Kármán eddies. Figure 4 illustrates $\lambda_{2}<0$ isocontours and $\left.N_{k} \in\right] 0 ; 1$ [ isolines, depicting vortex cores and sheardominated regions respectively. Note that $N_{k}$ is defined as follows (Truesdell 1954): $N_{k}=\left\|(1 / 2)\left(\nabla \boldsymbol{v}-{ }^{\mathrm{T}} \nabla \boldsymbol{v}\right)\right\|_{F} /\left\|(1 / 2)\left(\nabla \boldsymbol{v}+{ }^{\mathrm{T}} \nabla \boldsymbol{v}\right)\right\|_{F}$, where superscript $\mathrm{T}$ denotes the transposed operator. The operator $\|\cdot\|_{F}$, applied to a given matrix $\boldsymbol{M}$, refers to the Frobenius norm and is expressed as $\sqrt{\operatorname{trace}\left(\boldsymbol{M}^{\mathrm{T}} \boldsymbol{M}\right)}$. Figure 4(a) shows that the unsteady forcing induces local starting jet vortices (hereafter denoted control vortices) that split the shear layer, owing to their opposite sign vorticity. This promotes the generation of smaller-scale vortices which are driven by a characteristic time scale, defined as $1 / S t_{j e t}=0.25,16$ times smaller than the von Kármán shedding time scale $1 / S t \approx 4$. The von Kármán vortex feeding process (i.e. the supply of vorticity to the growing vortex via the separated shear layer) can therefore be considered as quasi-steady, such that the vortex shedding is not fundamentally altered. Nevertheless, the introduction 
(a)

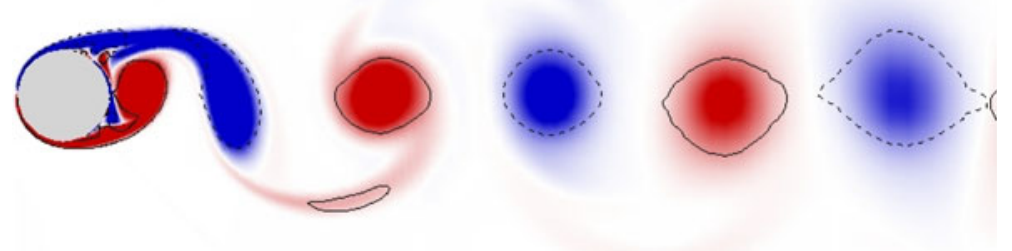

(b)

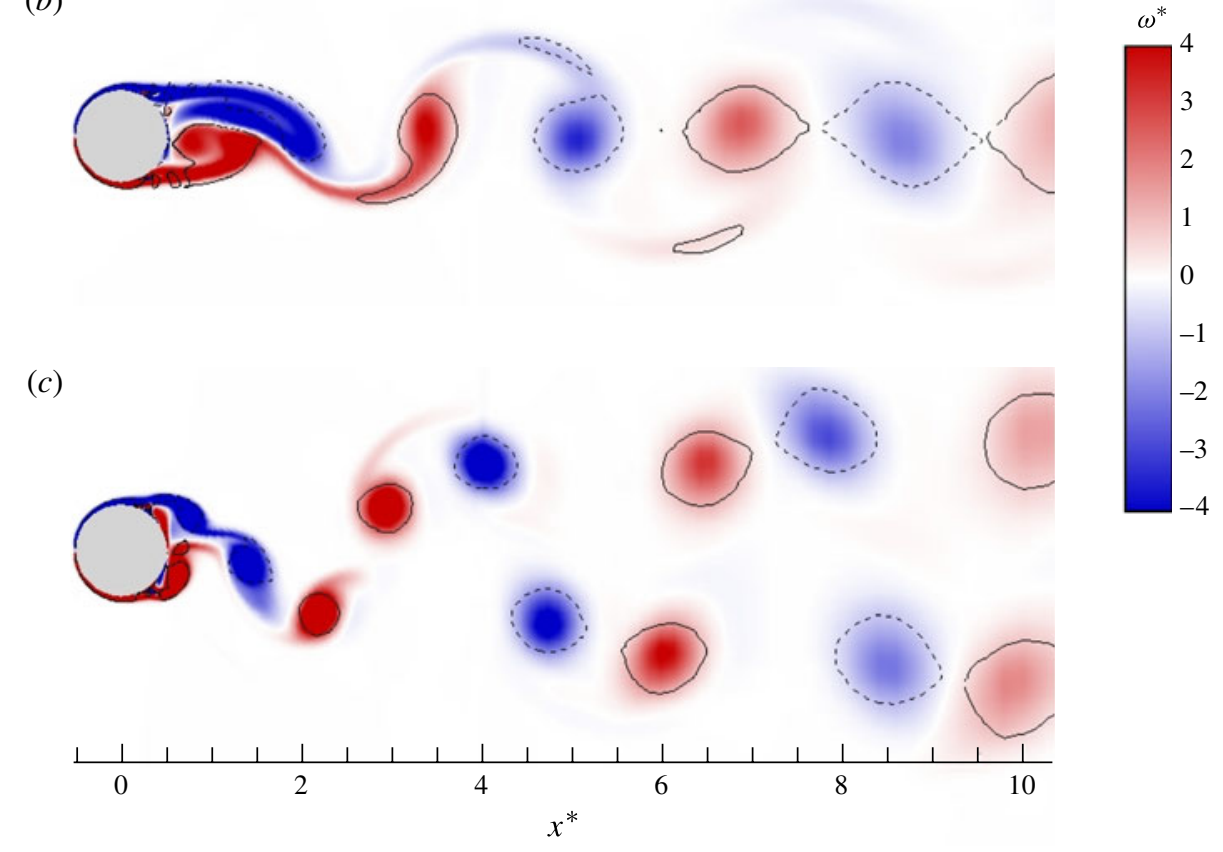

FIGURE 2. (Colour online available at journals.cambridge.org/flm) Normalized vorticity $\omega^{*}=\omega D / U_{\infty}$ computed for the unforced case $(a), S t_{\text {jet }}=4(b)$ and $S t_{\text {jet }}=0.5(c)$ forced cases. Dashed and solid lines are $\lambda_{2}=-1$ isolines identifying vortex cores with negative and positive vorticity respectively.

of opposite-sign vorticity inside the shear layer and the resulting splitting significantly affects the flow structure by drastically lowering the characteristic vorticity levels and circulation of the von Kármán vortices. Figure 3(a) indicates that the non-dimensional circulation $\Gamma^{*}$ (defined as $\Gamma^{*}=\Gamma / U_{\infty} D$ ) experienced by the von Kármán vortices for the $S t_{\text {jet }}=4$ forced case reaches an extremum of $\Gamma^{*}=2.2$ at $x^{*}=1.3$ downstream of the cylinder, whereas it saturates (i.e. it reaches a plateau) around $\Gamma^{*}=3.5$ between $x^{*}=0.8$ and $x^{*}=1.2$ for the unforced case. If one compares the vortex topologies of both the $S t_{j e t}=4$ forced and unforced case for a given phase following these extrema, it appears that the former depicts elongated von Kármán vortices confined to a narrower wake (see figure $2 b$ ). This strengthens the shear induced by the cross-wake interaction before the core vorticity saturation, observable on figure 3(a) through the progressive decrease of the vortices' circulation from $x^{*}=1.3$ to $x^{*}=2.1$, in contrast 

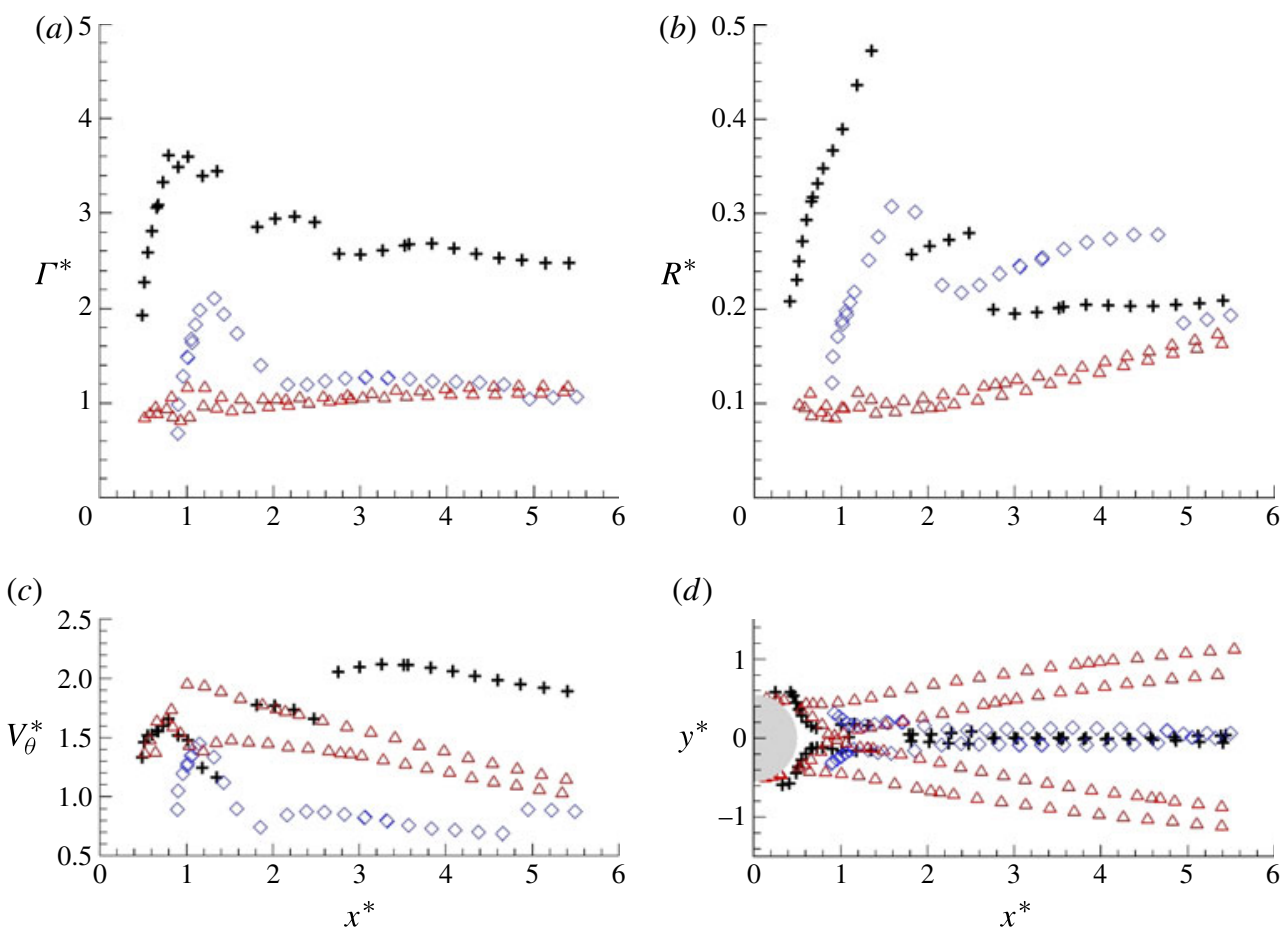

(d)

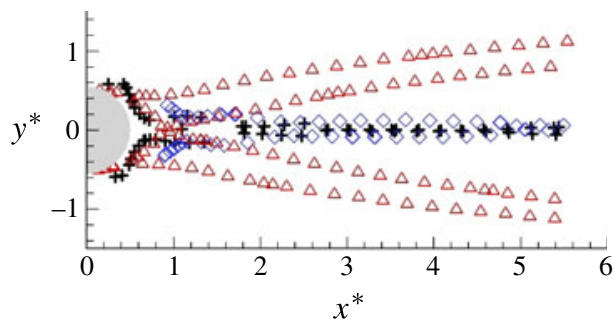

FIGURE 3. Normalized circulation $\Gamma^{*}=\Gamma / D U_{\infty}(a)$, radius $R^{*}=R / D(b)$, tangential velocity $V_{\theta}^{*}=V_{\theta} / U_{\infty}(c)$ and position $y^{*}=y / D(d)$ of the von Kármán vortices as a function of $x^{*}=x / D$. Symbols,$+ \diamond$ and $\Delta$ correspond to the unforced case, $S t_{j e t}=4$ and $S t_{j e t}=0.5$ forced cases respectively.

(a)

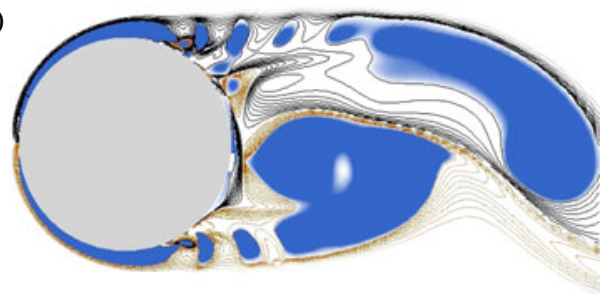

(b)

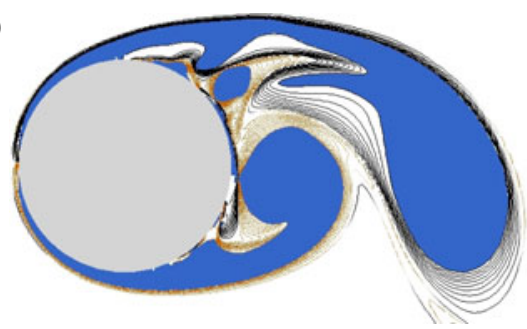

FIGURE 4. $\lambda_{2}<0$ isocontours (blue) and $\left.N_{k} \in\right] 0 ; 1[$ isolines coloured by vorticity (orange: positive, black: negative), depicting vortex cores and shear-dominated regions, respectively. $S t_{j e t}=4$ forced case $(a)$, natural case $(b)$.

with a more pronounced circulation drop for the unforced case at $x^{*}=1.3$. In addition, the sharp decrease of the von Kármán vortices radius (figure $3 b$ ), used as a quantitative criterion to identify this vortex shedding, occurs at $x^{*}=1.9$ and $x^{*}=1.3$ respectively. This shedding position delay observed for $S t_{j e t}=4$, the correlated time-averaged separation point delay and the vortices' circulation decrease significantly contribute to drag reduction, of the order of $60 \%$.

Further downstream the $S t_{j e t}=4$ forcing still impacts on the far-field wake structure as it delays the vortex-tail breakup, responsible for the second discontinuity observed 
(a)

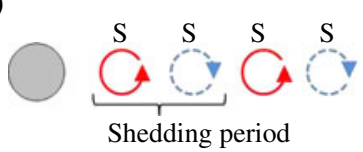

(b)

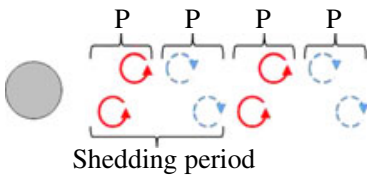

(c)

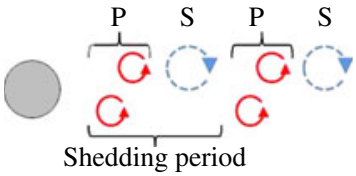

Figure 5. (Colour online) Schematic view of the $2 \mathrm{~S}(a), 2 \mathrm{P}(b)$ and $\mathrm{P}+\mathrm{S}(c)$ wake patterns.

as a drop in the vortex radius curve, from $x^{*}=2.5$ (unforced case) to $x^{*}=4.7$. Note that this vortex-tail breakup leads to a sudden increase of the vortex core azimuthal velocity, for both unforced and $S t_{j e t}=4$ forced cases (figure $3 c$ ). This process can be decomposed into two steps. First, the initially elongated shape of the vortex tail and its distance from the vortex core result in pronounced stretching (associated with local inertial effects) that leads to the separation from the vortex core. Second, the subsequent radius decrease strengthens the vortex core azimuthal velocity through angular momentum conservation.

\section{2. $S t_{j e t}=0.5$ forced flow}

In contrast, the $S t_{j e t}=0.5$ forcing considerably alters the shedding process since the von Kármán vortex feeding process can no longer be considered as quasi-steady with respect to the von Kármán shedding time scale.

The starting jet vortices produced by the actuators in the separated boundary layer periodically split the shear layer and force the shedding of counter-rotating vortex pairs such that the wake converges to a $2 \mathrm{P}$ pattern that locks on to a frequency of $S t_{j e t} / 2$. Such a pattern has previously been described by Williamson \& Roshko (1988), Jeon \& Gharib (2004) and Morse \& Williamson (2009) for oscillating cylinders. Figure $5(b)$ provides a schematic view of the $2 \mathrm{P}$ wake pattern. Each vortex pair is composed of two like-sign vortices respectively advected above and below the wake centreline, i.e. crossing the wake centreline or not, depending on whether their shedding occurs during the ascending or descending phase of the wake oscillation. The Lagrangian mapping of the vortex dynamics depicts four centroid trajectories that are significantly shifted away from the centreline in comparison to the unforced and $S t_{\text {jet }}=4$ forced cases (figure $3 d$ ).

Another prominent difference is the absence of a maximum in the circulation evolution (figure $3 a$ ), denoting the absence of saturation and cross-wake interaction. Here the vortices are dropped before they reach a sufficient strength and naturally move away from the cylinder. This clearly shows greater sensitivity of the feeding and shedding processes to this specific forcing frequency. A direct consequence is the cancellation of the vortex tail formation, as can be seen from the continuous evolution of the different vortex parameters in the wake of the cylinder (see figure 3 ).

Interestingly, notwithstanding these fundamental differences, the far-wake vortices' normalized circulation $\Gamma_{f w}^{*}$ of both the $S t_{j e t}=0.5$ and $S t_{j e t}=4$ forced cases converge to similar values, close to 1.1 (figure $3 a$ ).

This trend is confirmed for several cases in the quasi-steady regime, fixing $C_{j e t}$ and varying $\lambda_{\text {jet }}$ and $S t_{\text {jet }}$ (summarized in table 1). This is also observed for the lock-on regime, although this regime is more complex in that it may involve atypical $2 \mathrm{P}$ or $\mathrm{P}+\mathrm{S}$ wake patterns (see schematic view in figures $5 b$ and $5 c$ ). In particular it can be seen that the $2 \mathrm{P}$ pattern involves vortex pairs whose vortices have different circulations (cases 1 and 4), depending on their history, i.e. their shedding instant and initial trajectory. Such behaviour is further amplified in cases 7 and 10 where the 


\begin{tabular}{|c|c|c|c|c|c|c|c|}
\hline Case & $S t_{j e t}$ & $C_{j e t}$ & $\lambda_{\text {jet }}$ & $C_{D}$ & $\left|\Gamma_{f w}^{*}\right|$ & $S t$ & $\begin{array}{l}\text { Wake } \\
\text { pattern }\end{array}$ \\
\hline Ref. & - & - & - & 1.56 & 2.47 & 0.24 & $2 \mathrm{~S}$ \\
\hline 1 & 0.5 & 0.067 & 0.5 & 1.01 & $1.13 / 1.20$ & $0.25\left(S t_{i e t} / 2\right)$ & $2 \mathrm{P}$ \\
\hline 2 & 2 & 0.067 & 0.5 & 0.63 & 1.08 & 0.26 & $2 \mathrm{~S}$ \\
\hline 3 & 4 & 0.067 & 0.5 & 0.63 & 1.06 & 0.26 & $2 \mathrm{~S}$ \\
\hline 4 & 0.5 & 0.067 & 0.2 & 1.33 & $1.10 / 1.60$ & $0.25\left(S t_{i e t} / 2\right)$ & $2 \mathrm{P}$ \\
\hline 5 & 2 & 0.067 & 0.2 & 0.60 & 0.99 & 0.29 & $2 \mathrm{~S}$ \\
\hline 6 & 4 & 0.067 & 0.2 & 0.60 & 0.95 & 0.29 & $2 \mathrm{~S}$ \\
\hline 7 & 0.5 & 0.033 & 0.5 & 1.17 & $2.24 / 0.44 / 1.44$ & $0.25\left(S t_{j e t} / 2\right)$ & $\mathrm{P}+\mathrm{S}$ \\
\hline 8 & 2 & 0.033 & 0.5 & 1.07 & 1.95 & 0.26 & $2 \mathrm{~S}$ \\
\hline 9 & 4 & 0.033 & 0.5 & 1.06 & 1.99 & 0.26 & $2 \mathrm{~S}$ \\
\hline 10 & 0.5 & 0.033 & 0.2 & 1.20 & $2.18 / 0.66 / 1.72$ & $0.25\left(S t_{j e t} / 2\right)$ & $\mathrm{P}+\mathrm{S}$ \\
\hline 11 & 2 & 0.033 & 0.2 & 0.99 & 1.86 & 0.25 & $2 \mathrm{~S}$ \\
\hline 12 & 4 & 0.033 & 0.2 & 1.02 & 1.92 & 0.26 & $2 \mathrm{~S}$ \\
\hline
\end{tabular}

TABLE 1. Overall comparison of the influence of $S t_{j e t}, C_{j e t}$ and $\lambda_{\text {jet }}$ on the drag $C_{D}$, the farwake von Kármán vortices' circulation magnitude $\left|\Gamma_{f w}^{*}\right|$, the normalized frequency $S t$ of the vortex shedding and the wake topology. The first row corresponds to the unforced case. $2 \mathrm{P}$ and $\mathrm{P}+\mathrm{S}$ wake regimes may involve vortices of different circulation magnitude, depending on their history. For a given forced case, multiple values of circulation magnitude refer to these distinct vortices.

$\mathrm{P}+\mathrm{S}$ wake pattern is observed (note that the $\mathrm{P}+\mathrm{S}$ regime is critical as it represents a transition between $2 \mathrm{~S}$ and $2 \mathrm{P}$ wake regimes in the $\left(S t_{j e t}, C_{j e t}\right)$ phase space).

The overall comparison finally suggests that the intensity of the far-wake vortices is predominantly driven by the $C_{j e t}$ parameter through the introduction of opposite-sign vorticity in the shear layer. For instance, halving $C_{j e t}$ roughly increases $\Gamma_{f w}^{*}$ by a factor of 2. For a given flow regime, this results in the reduction of the drag coefficient of the cylinder $C_{D}$. Note that the drag reduction associated with the thrust of the jets (equal to $C_{j e t} \sin \left(\theta_{j e t}\right)$ ) is negligible in comparison to that associated with the wake modification. However the transition to atypical wake patterns $(2 \mathrm{P}$ or $\mathrm{P}+\mathrm{S})$, via $S t_{j e t}$, limits the drag reduction. As a consequence, the evolution of the drag reduction as a function of $S t_{\text {jet }}$ follows an asymptotic trend. This is in qualitative agreement with results obtained by Glezer, Amitay \& Honohan (2005) on the influence of normal-towall synthetic jets on the flow over a circular cylinder at $R e=7.55 \times 10^{4}$.

\section{Frequency-domain analysis}

The spatial analysis in the previous section revealed the sensitivity of the flow to $S t_{j e t}, C_{j e t}$ and $\lambda_{j e t}$, in terms of wake topology and vortex characteristics. However the coupling between the actuation modes, mainly driven by $S t_{j e t}$ and to a minor extent by $C_{j e t}$ and $\lambda_{j e t}$, and the primary instability of the unforced flow, is not easily approached. In this section we propose to apply DMD to discriminate between the different dynamic components of the flow, in order to get a better description of the physics.

The dynamic modes are computed using the spectral analysis described in $\S 3.1$. The flow-field database used for the calculation comprises $N=280$ snapshots spanning a shedding period. Note that $N$ is fixed large enough to ensure both spectral analysis convergence (Schmid 2010) and time resolution covering all relevant flow frequencies. 

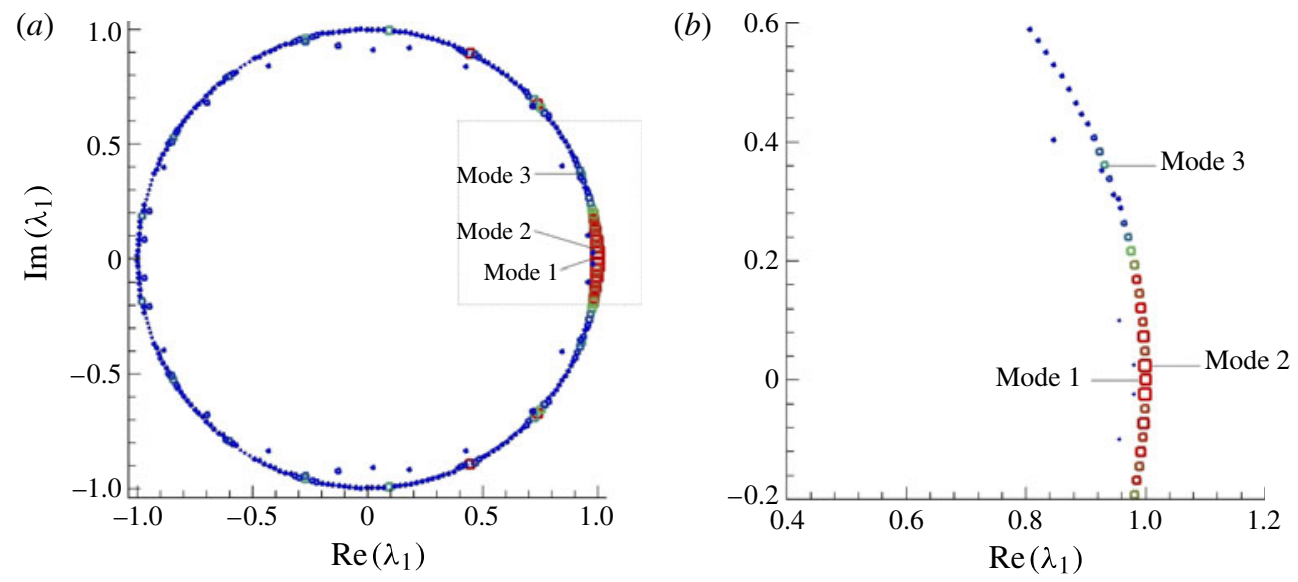

FIGURE 6. Imaginary versus real parts of the $S t_{j e t}=4$ forced case eigenvalues $\lambda_{i}$, coloured and sized by $\left\|\phi_{i}\right\|$. Hot colours and large symbols indicate large $\left\|\phi_{i}\right\|$. Close-up view of the full spectrum $(a)$ is displayed in $(b)$.

\subsubsection{Spectral analysis}

$$
\text { 5.1. } S t_{j e t}=4 \text { forced flow }
$$

Figure 6 displays the $\left(\operatorname{Re}\left(\lambda_{i}\right), \operatorname{Im}\left(\lambda_{i}\right)\right)$ spectrum computed from the $y$-velocity component of the $S t_{j e t}=4$ forced flow. Spectral analysis of the $x$-velocity component gives similar results and therefore is not presented here. One can notice that eigenvalues lie on the unit circle $\left|\lambda_{i}\right|=1$, indicating that the system evolves on an attractor (Mezić 2005).

Figure 7 depicts the real part of the associated dynamic modes. The imaginary part reflects the characteristic wavenumber through a $90^{\circ}$ phase offset of the real part and is not illustrated here. The three fundamental modes correspond to the mean flow field (mode 1), the von Kármán street mode (mode 2) and the actuation mode (mode 3). They are associated with eigenvalues $\lambda_{k}$ via Strouhal numbers $S t_{k}=\omega_{k} \times D / U_{\infty}=\operatorname{Im}\left\{\log \left(\lambda_{k}\right)\right\} \times D /\left(2 \pi U_{\infty} \Delta t\right)$. Here $S t_{1}=0, S t_{2}=0.26$ and $S t_{3}=4$, respectively.

The most prominent result is the train of counter-rotating vortices revealed by mode 3 (figures $7 c$ and $7 d$ ) as successive opposite-sign contours of $y$-velocity modal components. This scheme closely resembles the flow induced by a plasma dielectric barrier discharge actuator in a stagnant medium (private communication, Y. Bury \& P. Galtier, 2010, technical note on the characterization of the flow induced by a DBD actuator through time-resolved PIV). Since the spatial analysis only depicts co-rotating control vortices separated by shear zones (figure 4), DMD hence proves its ability, through the actuation mode, to isolate the dynamics of the actuator in interaction with its environment. This point is detailed hereafter on the basis of time-reconstructed data. In addition, one can observe that the levels of mode $3 y$-velocity components progressively decay as associated small- scale control structures are advected along the shear layer, circumscribing the mean separated region observable in mode 1 (figure $7 a$ ). It is also noteworthy that the signature of these small-scale modal structures still persists further downstream from the separated region.

Similarly, large-scale and lower-frequency modal structures are captured by mode 2 (figure $7 b$ ). This mode is unstable as revealed by its positive real part and corresponds to the primary von Kármán instability. 
(a)

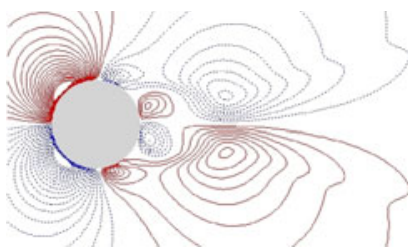

(c)

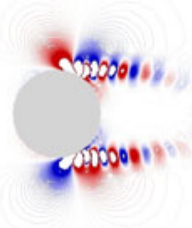

(b)

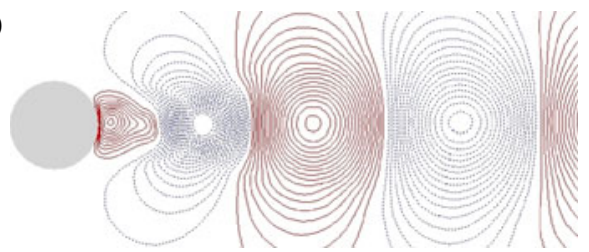

(d)

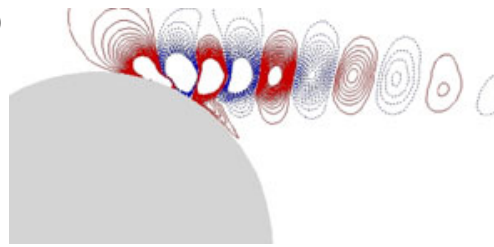

FIGURE 7. (Colour online) Isolines of the $y$-velocity modal structures obtained for the $S t_{j e t}=4$ forced case (solid lines: positive, dotted lines: negative). Mode $1(a)$, mode $2(b)$, mode $3(c)$, and close-up view of mode $3(d)$.

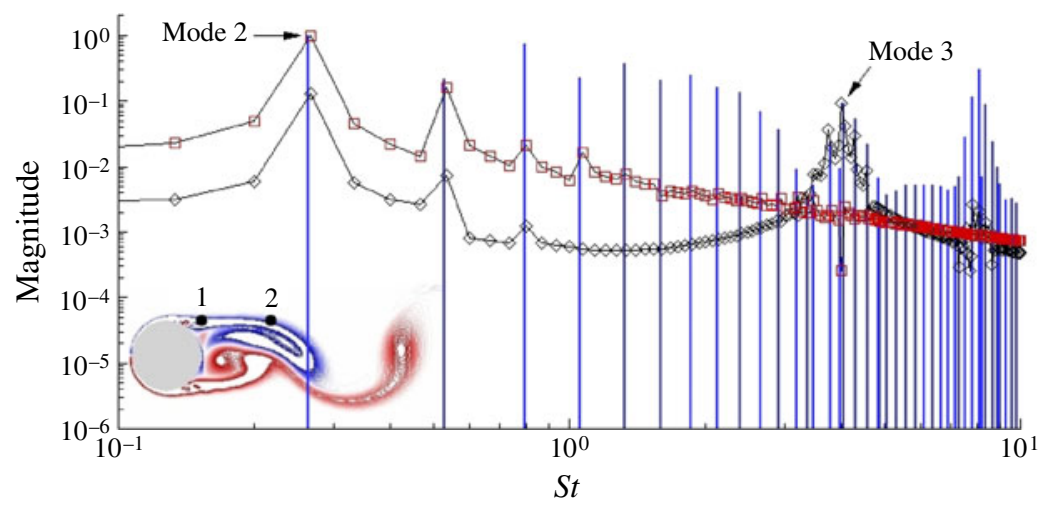

FIGURE 8. (Colour online) $y$-velocity mode magnitude versus normalized frequency $S t$ (bars). Symbols $\diamond$ and $\square$ correspond to fast Fourier transforms computed from the $y$-velocity time signals extracted from probe locations 1 and 2 respectively (see inset picture). $S t_{j e t}=4$ forced case.

Modes 2 and 3 are fundamentals of harmonic spectra $\zeta^{2}$ and $\zeta^{3}$ intrinsically induced by non-strictly sinusoidal time-varying Eulerian data. Corresponding harmonics are revealed on figure 8 by plotting the modes' magnitude versus normalized frequency $S t$. Moreover, the coupling between these two harmonic spectra leads to additional modes. For instance, interactions between the fundamentals and harmonics of modes 2 and 3 generate beating frequencies $n S t_{3} \pm m S t_{2}$, where $m$ and $n$ are positive integers.

Power spectra derived from temporal signals at various locations in the vicinity of the cylinder corroborate these results. Figure 8 reveals that probes located in the shear layer and in the wake partly capture the harmonic spectra resulting from the tangential jet actuation and the von Kármán vortex shedding.

\subsubsection{Time reconstruction}

The spectral-domain analysis identified each of the harmonic spectra $\zeta^{k}$ associated with fundamental dynamic modes $k$. These spectra enable time reconstruction of each 

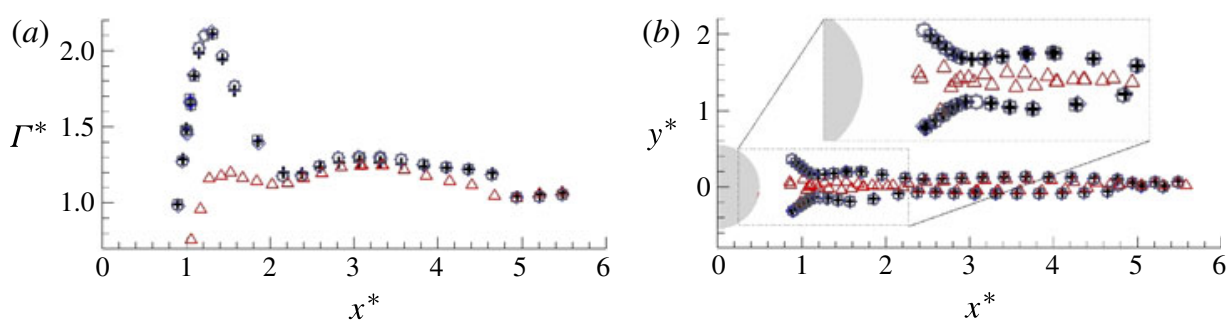

FIgURE 9. (Colour online) Normalized circulation $\Gamma^{*}=\Gamma / D U_{\infty}(a)$ and position $y^{*}=y / D$ $(b)$ of the raw and time-reconstructed von Kármán vortices as a function of $x^{*}=x / D$. Symbols $+, \Delta, \diamond$ and $\square$, correspond to the raw data and the $\zeta^{2}, \zeta^{1}+\zeta^{2}$ and $\zeta^{1}+\zeta^{2}+\zeta^{3}$ harmonic-spectra-based time reconstructions respectively. $S t_{j e t}=4$ forced case.

frequency component of the flow, through relation (3.3), where $i$ indexes any of the $\zeta^{k}$ harmonic components. In this context, vortex tracking is applied on the basis of these frequency band reconstructions.

Following this idea, figure 9 compares the relevant parameters of the coherent structures based on the $\zeta^{2}, \zeta^{1}+\zeta^{2}$ and $\zeta^{1}+\zeta^{2}+\zeta^{3}$ harmonic spectra-based time reconstructions with those obtained from the spatial-domain analysis of the instantaneous flow fields.

The $\zeta^{2}$ harmonic-spectrum-based reconstruction closely matches the dynamics of the von Kármán vortices in the far-wake zone (for $x^{*}>4.7$ ), i.e. beyond the vortex-tail breakup, as can be seen in figure 9. However the occurrence of the von Kármán vortex shedding and the vortex-tail breakup is not revealed. In fact, the associated sharp discontinuities in the spatio-temporal evolution of the vortex parameters can be retrieved by taking into account the mean component of the flow, that is to say by reconstructing upon $\zeta^{1}+\zeta^{2}$ harmonic spectra.

On the other hand, the addition of $\zeta^{3}$ to $\zeta^{1}+\zeta^{2}$ harmonic spectra reconstruction does not fundamentally alter the large-scale structure evolution. This is highlighted by the fitting of both $\zeta^{1}+\zeta^{2}$ and $\zeta^{1}+\zeta^{2}+\zeta^{3}$ curves (figure 9) and this suggests a weak coupling between the primary instability and the actuation. As previously mentioned, this can be related to the localized action of control vortices that is essentially confined to shear layer splitting and does not fundamentally alter the von Kármán vortex dynamics. Finally it is shown that the remaining harmonic spectra related to the beating frequencies have a negligible influence on the time-reconstructed fields.

To complete the discussion regarding the $S t_{\text {jet }}=4$ forced flow it is worth considering the number of harmonics needed to ensure convergence of the timereconstructed fields associated with the $\zeta^{2}$ and $\zeta^{3}$ harmonic spectra. Whereas $\zeta^{2}$-based time reconstruction requires the use of the fundamental mode and its five following harmonics (i.e. $i=1-6$ ), $\zeta^{3}$-based time reconstruction convergence only requires the fundamental mode $(i=1)$. This is explained by the fact that imposed forcing fluctuations induce Eulerian signals close to a sine function.

\section{2. $S t_{\text {jet }}=0.5$ forced flow}

\subsubsection{Spectral analysis}

The same analysis is performed for the $S t_{j e t}=0.5$ forced flow. As expected, the $\left(\operatorname{Re}\left(\lambda_{i}\right), \operatorname{Im}\left(\lambda_{i}\right)\right)$ spectrum is concentrated on an attractor set. The spectrum reveals two dominant modes, corresponding to the mean flow field (mode 1) and the primary instability mode (mode 2) respectively. In this particular case, the actuation mode, 
(a)

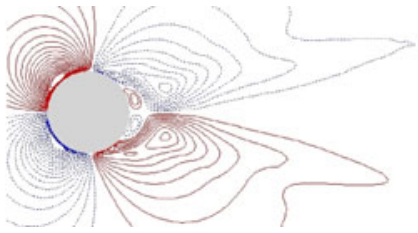

(c)

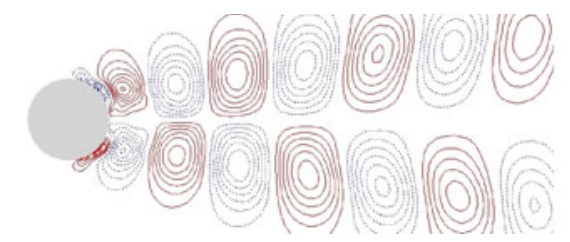

(b)

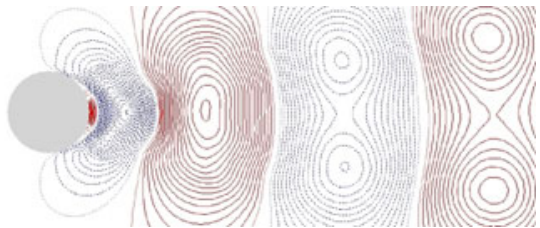

(d)

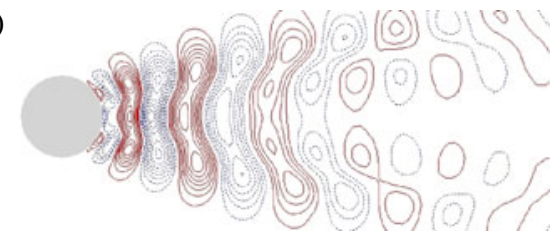

FIGURE 10. (Colour online) Isolines of the $y$-velocity modal structures obtained for $S t_{\text {jet }}=0.5$ forced case (solid lines: positive, dotted lines: negative). Mode $1(a)$, mode 2 $(b)$, mode $3(c)$ and mode $4(d)$.

characterized by a Strouhal number of 0.5 , merges with the first harmonics of mode 2. This highlights the so-called lock-on phenomenon, which leads to the periodic shedding of counter-rotating vortex pairs at $S t_{2}=0.25$. The resulting $S t_{3}=0.5$ dynamic mode will hereafter be referred to as mode 3 .

Figure 10 depicts the real part of the $y$-velocity modal structures associated with mode 1 , mode 2 , mode 3 and mode 4 . Mode 2 reveals the $2 \mathrm{P}$ pattern through the advection of counter-rotating vortex pairs in the wake of the cylinder. Each vortex pair comprises two like-sign structures symmetrically distributed with respect to the $y$-velocity component, on both sides of the wake centreline. A distinctive feature is the asymmetry of mode 3 characterized by the $180^{\circ}$ phase shift of the structures located above the wake centreline with respect to the structures located below. This phase shift suggests that mode 3 carries the signature of the vortex pair tilting, with respect to the $y$-axis, as previously illustrated on figure 2 (see $\S 4$ ). This point is detailed in the next subsection.

\subsubsection{Time reconstruction}

In this particular case dominated by the lock-on phenomenon, the spectraldomain analysis identified two distinct harmonic spectra $\zeta^{1}$ and $\zeta^{2}$, associated with fundamental dynamic modes 1 and 2 as previously introduced. In order to determine the role of each of the $\zeta^{2}$ harmonics in the flow-field topology, figure 11 shows the spectra-based time reconstructions, in terms of vorticity, obtained from relation (3.3). In addition, figure 12 compares the relevant vortex parameters computed from the successive spectra-based time reconstructions with those from the spatial-domain analysis of the instantaneous flow fields.

As expected, time reconstruction upon mode 2 restores a strictly symmetric flow field structure with respect to the $x$-axis (figure $11 a$ ). The shedding process of the counter-rotating vortex pairs is properly recovered without, however, distinguishing the trajectories of like-sign vortices advected in both the $y>0$ and $y<0$ regions, crossing the wake centreline or not. The dynamics of each vortex of a given pair is actually recovered by mode 3 . Indeed, the latter is associated with phenomena that occur at $S t_{3}=0.5$, i.e. with the consecutive shedding of counter-rotating vortices. Therefore mode 3 introduces a $1 / S t_{3}$ time shift between the two vortices of a vortex pair. This time shift inherently yields to the vortex pair tilting with respect to the $y$-axis (figure 11b). Eventually the precise vortices trajectories are fully recovered 
(a)

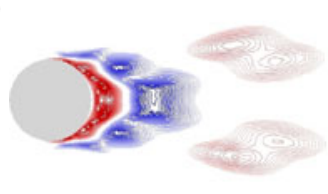

(c)

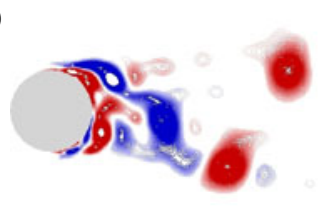

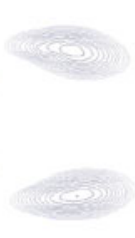

(.) (b)

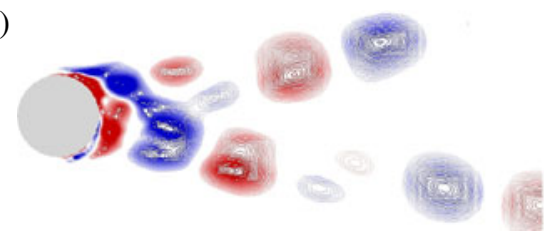

(d)

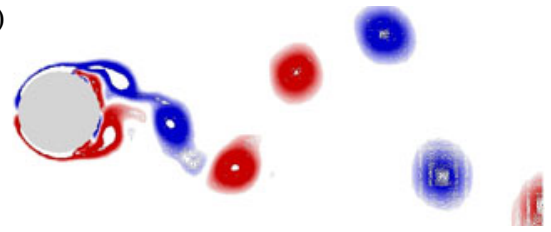

FIGURE 11. (Colour online) Isolines of the time-reconstructed normalized vorticity fields based on $\zeta^{2}$ harmonic spectrum fundamental mode $(a), \zeta^{2}$ first two harmonics $(b), \zeta^{2}$ first six harmonics $(c), \zeta^{2}$ first six harmonics and $\zeta^{1}$ harmonic spectrum $(d) . S t_{j e t}=0.5$ forced case.
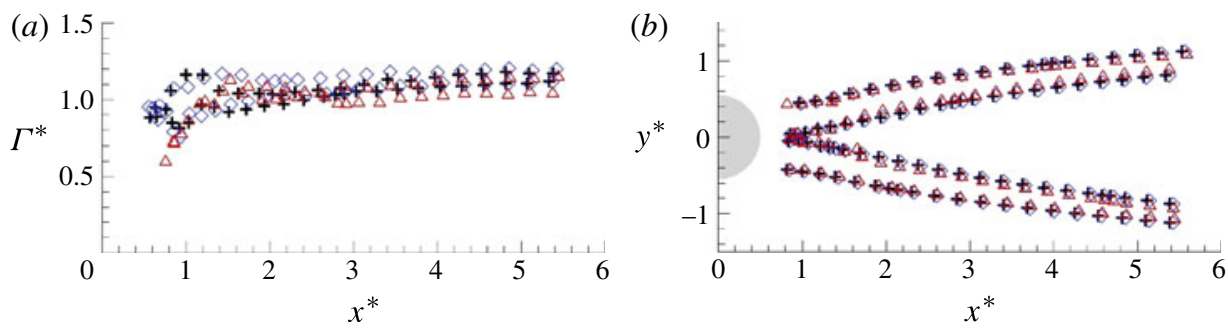

FIgURE 12. (Colour online) Normalized circulation $\Gamma^{*}=\Gamma / D U_{\infty}(a)$ and position $y^{*}=$ $y / D(b)$ of the raw and time-reconstructed von Kármán vortices as a function of $x^{*}=x / D$. Symbols,$+ \Delta$ and $\diamond$ correspond to the raw data, $\zeta^{2}$ and $\zeta^{1}+\zeta^{2}$ harmonic-spectra-based time reconstructions respectively. $S t_{j e t}=0.5$ forced case.

using the $\zeta^{2}$ fundamental mode and its three following harmonics (i.e. $\left.i=1-4\right)$ and the $\zeta^{1}$ fundamental mode (mean flow). Yet recovering the precise intensity and radius requires the $\zeta^{2}$ fundamental mode and its nine following harmonics (i.e. $i=1-10$ ) in addition to the mean flow field (figures $11 d$ and 12).

\section{Conclusion}

Pulsed jets have proved to be a relevant flow control strategy in a variety of applications, such as pressure drag reduction, stabilization of slender forebodies, airframe noise reduction and gas mixing in nozzle jets. Their implementation in massively separated flow configurations is primarily motivated by their ability to modify relevant flow frequencies, potentially leading to a substantial modification of the wake structure, affecting in particular the vortical activity.

We numerically investigated the effect of pulsed tangential jets on the vortical dynamics in the vicinity of a two-dimensional circular cylinder at Reynolds number 1000 , varying the normalized actuation frequency $S t_{j e t}$, momentum coefficient $C_{j e t}$ and duty cycle $\lambda_{j e t}$. Time-series data were analysed using a spectral-Lagrangian dual approach based on dynamic mode decomposition (DMD) and vortex tracking.

Through this analysis, it is shown that the flow response to the actuation is driven by the strong interaction between shear layers and pulsed jets. The latter preferentially lead to the lock-on regime or to the quasi-steady vortex feeding regime, whether 
the excitation frequency is of the order of, or significantly greater than, the natural instability frequency. We focused on two distinct cases, typical of each regime, associated with $S t_{j e t}=0.5$ and $S t_{j e t}=4$ respectively.

In the first case, we show that the wake topology is dramatically altered and locks onto a $2 \mathrm{P}$ wake regime. This is reflected in four periodically shed vortices that are significantly shifted away from the wake centreline. Their shedding occurs before vorticity saturation, avoiding vortex tail formation and leading to continuous evolution of vortex core parameters, as opposed to the unforced case. The modal decomposition emphasizes the strong coupling between the natural instability and the actuation frequency through a unique harmonic spectrum, where the actuation frequency merges with the first harmonics of the fundamental vortex shedding frequency. Interestingly, time reconstruction over those two first harmonics recovers the dynamics of the flow associated with the $2 \mathrm{P}$ pattern and vortex pair tilting.

In contrast the $S t_{j e t}=4$ forced case converges to a more common von Kármán street mode. It is characterized by two distinct harmonic spectra, associated with the vortex shedding instability and the actuation mode respectively. This indicates a weak coupling between the forcing and shedding frequencies. DMD retrieved the rotational contribution associated with these two specific time scales, through modalbased time reconstruction upon each given spectrum. This emphasizes the underlying physics driving the flow associated with either the actuator or the vortex shedding instability. The Lagrangian signature of these two modes demonstrates small-scale actuator-induced vortices that remain localized in the shear layers which are split under a quasi-steady process. This results in a decrease of the intensity of the von Kármán vortices through the introduction of opposite-sign vorticity in the shear layers.

Finally the complementary study on the impact of the $S t_{j e t}, C_{j e t}$ and $\lambda_{\text {jet }}$ parameters reveals that the intensity of the von Kármán vortices is predominantly driven by $C_{j e t}$. However the influence of the circulation attenuation on drag reduction may partly be counterbalanced by a deep alteration of the wake topology (which increases pressure drag), under the influence of $S t_{j e t}$, potentially leading to $2 \mathrm{P}$ or $\mathrm{P}+\mathrm{S}$ wake regimes.

Such wake patterns reveal remarkable topological analogies with oscillating cylinders. The problem is different though, since cylinder oscillations imply in particular a global modification of the flow conditions on the whole surface of the cylinder, the quasi-cancellation of forcing-induced vorticity due to the reciprocating motion as well as added mass effects.

As a conclusion, the spectral-Lagrangian dual approach provides insight into the interaction mechanisms (between the tangential jets and the separated shear layers) governing the forced flow past a circular cylinder. This deeper knowledge could provide key features for the definition of effective flow control strategies. Substantial benefits could be obtained regarding drag reduction, noise control and mixing, through the modification of Strouhal number $S t$ and the wake pattern.

\section{Acknowledgement}

The authors gratefully acknowledge the French Ministry of Defence and the DGA for supporting this work.

\section{REFERENCES}

Bury, Y., Morton, S. A. \& Charles, R. 2008 Experimental investigation of the flow field in the close wake of a simplified C-130 shape: a model approach of airflow influence on airdrop. 26th AIAA Applied Aerodynamics Conference, Honolulu, Hawaii, USA. 
Choi, H., Jeon, W. P. \& Kim, J. 2008 Control of flow over a bluff body. Annu. Rev. Fluid Mech. 40, 113-139.

GERRARD, J. H. 1966 The mechanics of the vortex formation region of vortices behind bluff bodies. J. Fluid Mech. 25, 401-413.

Glezer, A. \& Amitay, M. 2002 Synthetic jets. Annu. Rev. Fluid Mech. 34, 503-529.

Glezer, A., Amitay, M. \& Honohan, A. M. 2005 Aspect of low- and high-frequency actuation for aerodynamic flow control. AIAA J. 43, 1501-1511.

HENDERSON, R. D. 1997 Nonlinear dynamics and pattern formation in turbulent wake transition. J. Fluid Mech. 352, 65-112.

JEON, D. \& GHARIB, M. 2004 On the relationship between the vortex formation process and cylinder wake vortex patterns. J. Fluid Mech. 519, 161-181.

JeOng, J. \& Hussain, F. 1995 On the identification of a vortex. J. Fluid Mech. 285, 69-94.

JuKES, T. N. \& CHOI, K. S. 2009 Control of unsteady flow separation over a circular cylinder using dielectric-barrier-discharge surface plasma. Phys. Fluids 21, 094106.

Liu, F., Luo, S., Gao, C., Meng, X., HaO, J., Wang, J. \& ZhaO, Z. 2008 Flow control over a conical forebody using duty-cycled plasma actuators. AIAA J. 46, 2969-2973.

MEZIĆ, I. 2005 Spectral properties of dynamical systems, model reduction and decompositions. Nonlinear Dyn. 41, 309-325.

Morse, T. L. \& Williamson, C. H. K. 2009 Prediction of vortex-induced vibration response by employing controlled motion. J. Fluid Mech. 634, 5-39.

Post, M. L. \& CORKE, T. C. 2004 Separation control on high angle of attack airfoil using plasma actuators. AIAA J. 42, 2177-2184.

Rowley, C. W., Mezić, I., Bagheri, S., Schlatter, P. \& Henningson, D. S. 2009 Spectral analysis of nonlinear flows. J. Fluid Mech. 641, 115-127.

SCHMID, P. J. 2009 Dynamic mode decomposition of experimental data. 8th International Symposium on Particle Image Velocimetry, Melbourne, Victoria, Australia.

SCHMID, P. J. 2010 Dynamic mode decomposition of numerical and experimental data. J. Fluid Mech. 656, 5-28.

Schmid, P. J., MeYer, K. E. \& Pust, O. 2009 Dynamic mode decomposition and proper orthogonal decomposition of flow in a lid-driven cylindrical cavity. 8th International Symposium on Particle Image Velocimetry, Melbourne, Victoria, Australia.

SCHMid, P. J. \& Sesterhenn, J. 2008 Dynamic mode decomposition of numerical and experimental data. Sixty-First Annual Meeting of the APS Division of Fluid Dynamics, San Antonio, Texas, USA.

Schmid, P. J., Violato, D., Pust, O. \& Scarano, F. 2010 Decomposition of time-resolved tomographic PIV. In 15th International Symposium on Applications of Laser Techniques to Fluid Mechanics, Lisbon, Portugal.

Thomas, F. O., Kozlov, A. \& CoRke, T. C. 2008 Plasma actuators for cylinder flow control and noise reduction. AIAA J. 46, 1921-1931.

Truesdell, C. 1954 The Kinematics of Vorticity. Science Series, vol. 19, Indiana University Publications.

Williamson, C. H. K. 1996 Vortex dynamics in the cylinder wake. Annu. Rev. Fluid Mech. 28, 477-539.

Williamson, C. H. K. \& RoshKo, A. 1988 Vortex formation in the wake of an oscillating cylinder. J. Fluids Struct. 2, 355-381. 